\title{
Feasibility of Quantum Communications in Aquatic Scenario
}

\author{
Tarantino, Silvia; Cozzolino, Daniele; Rottwitt, Karsten; Bacco, Davide
}

Published in:

Proceedings of 2018 IEEE Photonics Conference

Link to article, DOI:

10.1109/IPCon.2018.8527325

Publication date:

2018

Document Version

Peer reviewed version

Link back to DTU Orbit

Citation $(A P A)$ :

Tarantino, S., Cozzolino, D., Rottwitt, K., \& Bacco, D. (2018). Feasibility of Quantum Communications in Aquatic Scenario. In Proceedings of 2018 IEEE Photonics Conference (pp. 2 pp.). IEEE.

https://doi.org/10.1109//PCon.2018.8527325

\section{General rights}

Copyright and moral rights for the publications made accessible in the public portal are retained by the authors and/or other copyright owners and it is a condition of accessing publications that users recognise and abide by the legal requirements associated with these rights.

- Users may download and print one copy of any publication from the public portal for the purpose of private study or research.

- You may not further distribute the material or use it for any profit-making activity or commercial gain

- You may freely distribute the URL identifying the publication in the public portal

If you believe that this document breaches copyright please contact us providing details, and we will remove access to the work immediately and investigate your claim. 


\section{Feasibility of Quantum Communications in aquatic scenario}

\author{
${ }^{1}$ Silvia Tarantino \\ s161176@student.dtu.dk
}

\author{
${ }^{1}$ Daniele Cozzolino \\ dacoz@fotonik.dtu.dk
}

${ }^{1}$ Karsten Rottwitt

karo@fotonik.dtu.dk

\author{
${ }^{1}$ Davide Bacco \\ dabac@fotonik.dtu.dk
}

${ }^{1}$ CoE SPOC, DTU Fotonik, Department Photonics Eng., Technical University of Denmark, Ørsteds Plads 340, Kgs. Lyngby, 2800 Denmark

\begin{abstract}
Security in underwater communications is a very sensitive topic due to its great interest in scientific, industrial and military applications. We present a feasibility analysis of different types of quantum communications protocols in aquatic scenarios.
\end{abstract}

Index Terms-quantum cryptography, underwater communication, free-space optical communication

\section{INTRODUCTION}

The increasing number of vehicles sailing on the sea surface, or in the water medium itself, requests a secure and trustable channel to exchange information. Optical communications represent a solution for such an expanding demand, due to the achievable high data rate [1]. Moreover, to guarantee the ultimate security in the communication link, quantum communication is the most powerful resource [2]-[4]. In particular, quantum key distribution (QKD), a technique based on quantum physics, provides unconditional secure communications. This technology has already been demonstrated in optical fibers and free-space links, but only few proof-ofconcept experiments have been reported for the underwater environment [5]-[8]. Here we present a feasibility study of quantum communication protocols for multiples aquatic scenarios.

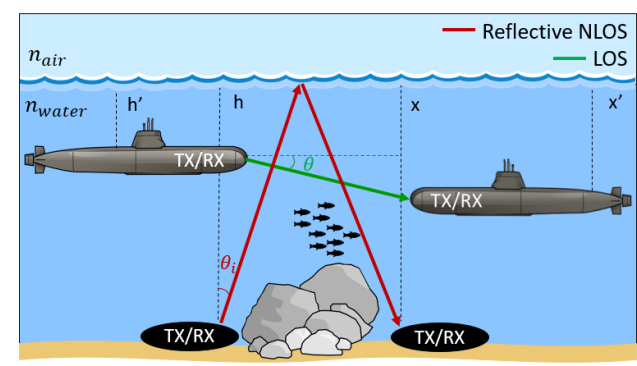

Fig. 1. Underwater link scenarios. Line of sight (LOS) underwater communication between moving vehicles. Non-line-of-sight (NLOS) underwater communications for sensors on the seabed.

\section{UNDERWATER OPTICAL CHANNEL}

The water can be used as an optical communication link for a specific wavelength range (green-blue) with a good transmittance value [1]. The attenuation of the signal is due to scattering and absorption, which are dependent on the selected wavelength [9]. We here consider the two optical channels showed in Figure 1. The line-of-sight (LOS) link, where the beam can directly travel from the transmitter (Alice) to the receiver (Bob), provides a reliable point-to-point connection. The transmissivity for the LOS link is defined in [1] as:

$$
t_{L O S}=\exp \left[-\frac{c \cdot d}{\cos \theta}\right]
$$

where $c=0.1514 / \mathrm{m}$ is the extinction coefficient at $532 \mathrm{~nm}$ for clear-ocean water, $\theta$ is the angle between the perpendicular to Bob plane and the transmitter-receiver trajectory and $d$ is the perpendicular distance between Alice and Bob's plane. In case of obstacles in the point-point connection, it is possible to use Snell's law between the air-water surface to totally reflect the optical beam. This type of link is defined as reflective non-line-of-sight (NLOS) link [1]. The transmissivity for the NLOS link is defined as a function of the angle $\theta_{i}$, see Fig. 1.

$$
t_{N L O S}=\left\{\begin{aligned}
\operatorname{Pr} \exp \left[-c \frac{x+h}{\cos \theta_{i}}\right] & \theta_{i}<\theta_{c} \\
\operatorname{Pexp}\left[-c \frac{x+h}{\cos \theta_{i}}\right] & \theta_{i} \geq \theta_{c} .
\end{aligned}\right.
$$

$P$ is the probability density function describing the wavy surface of the ocean (a Gram-Charlier series of approximate Gaussian distributions [1]), and $r$ represents the reflectance as a function of the incidence angle $\theta_{i}$. Only in the case of $\theta_{c} \geq 48.44$ total internal reflection (TIR) of the light occurs.

\section{QUANTUM COMMUNICATIONS PROTOCOLS}

Quantum states can be encoded in different degrees of freedom: polarization, phase, time and space [2]. Polarization and space, as degrees of freedom, have been demonstrated for a few meters link in in underwater environment [5]-[8], but a longer propagation distance will be affected by degradation of the signals (depolarization and broadening of the pulse) [7]. On the contrary, phase and time as modulation techniques lead to better performances for underwater optical wireless communications [9]

\section{RESUlts AND Discussion}

In a QKD system the main parameter is represented by the secret key rate, defined as [2]:

$$
K=R\left[I(A: B)-\min \left\{I_{A E}, I_{B E}\right\}\right]
$$




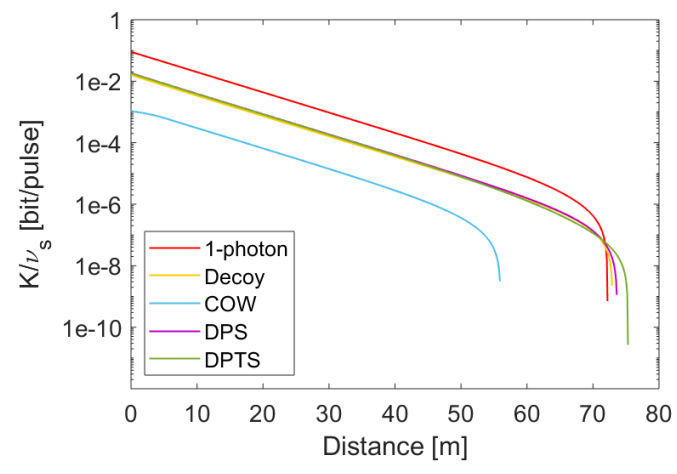

Fig. 2. LOS secret key rate as a function of the distance for different QKD protocols. Parameters used are reported in Table .

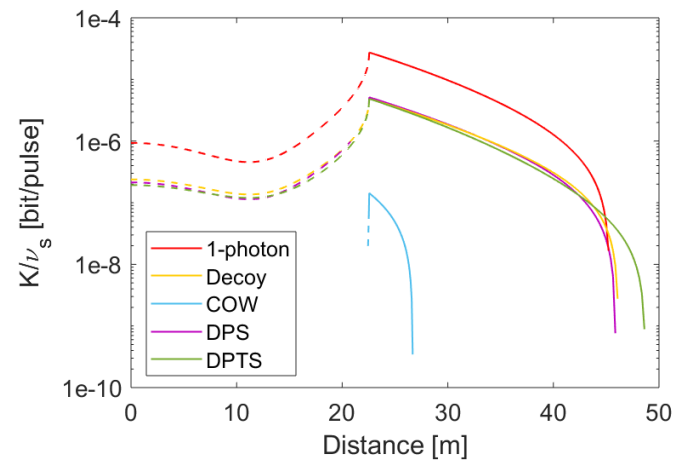

Fig. 3. NLOS secret key rate as a function of the distance for different QKD protocols. Dashed lines are key generations under the condition $\theta_{i}<\theta_{c}$. Full lines under the condition of TIR, i.e. unconditionally secure communications. Parameters used are reported in Table I.

where $R=\nu_{s} \eta t_{B} t$ is the raw key rate, $\nu_{s}$ is transmitter repetition rate, $\eta$ the detector's efficiency, $t_{B}$ the losses in Bob's apparatus and $t$ is the transmissivity for LOS or NLOS link. The mutual information between Alice and Bob, $I(A: B)=1-h(Q)$, is a function of $h(Q)$, the binary entropy of the quantum bit error rate (QBER). Finally, $I_{A E}$ and $I_{B E}$ indicate the information available to the eavesdropper Eve. The results for the simulations are shown in Fig. 2 and 3 for LOS and NLOS, respectively. In the case of the NLOS link, the condition of total internal reflection must be fulfilled in order to guarantee unconditional security. If $\theta_{i}<\theta_{c}$ a fraction of the incident beam is refracted on the sea surface and the eavesdropper Eve could potentially intercept the refracted ray. These results indicate that secure communications using quantum protocols can be obtained for several meters in a LOS link with Alice and Bob located $10 \mathrm{~m}$ below the sea level. In the case of a NLOS link, nevertheless a lower achievable distance is reported for sensors located at $10 \mathrm{~m}$ depth, a positive secret key rate can be achieved. In addition, Fig. 4 shows how the secret key rate varies under different windslope values. Even in the condition of a wavy ocean surface, a secret key could be extracted and therefore secure quantum communications implemented between.
TABLE I

PARAMETERS USED FOR SIMULATION IN FigURE 2 AND Figure 3.

\begin{tabular}{|c|c|}
\hline Parameter & Specification \\
\hline Transmitter/Receiver depth & $10[\mathrm{~m}]$ \\
\hline Upwind/downwind slope (wind speed $10.2 \mathrm{~m} / \mathrm{s}$ ) & $0\left[{ }^{\circ}\right]$ \\
\hline Water extinction coefficient & $0.1514\left[\mathrm{~m}^{-1}\right]$ \\
\hline Transmitted beam wavelength & $532[\mathrm{~nm}]$ \\
\hline Visibility and Dark-count probability & 0.99 and $10^{-7}$ \\
\hline
\end{tabular}

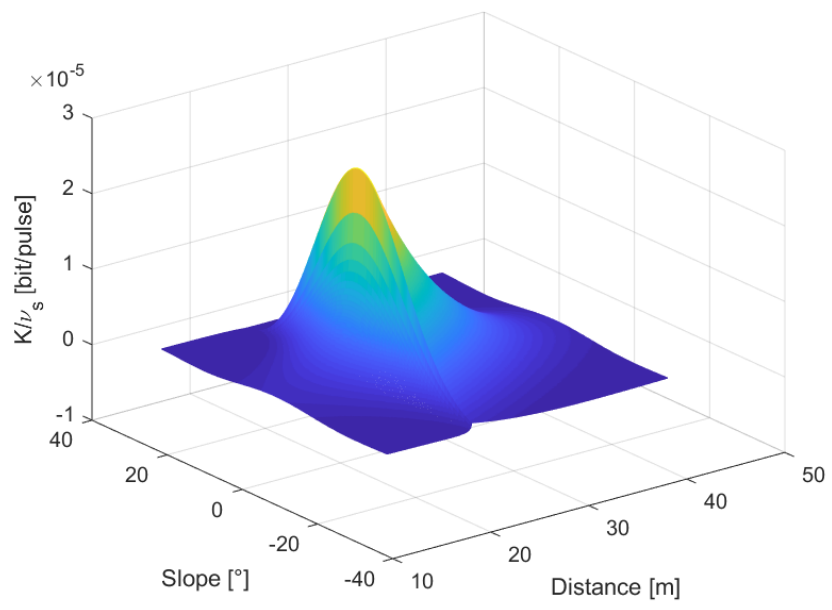

Fig. 4. Secret key rate for NLOS configuration as a function of the upwind/downwind slope. Parameter are reported in Table I.

\section{CONCLUSION}

A feasibility study of multiples QKD protocols under LOS and NLOS link scenarios is presented. This analysis proves the usefulness of quantum communications in the underwater environment, broadening the field of quantum technology.

\section{ACKNOWLEDGMENT}

Center of Excellence, SPOC-Silicon Photonics for Optical Communications (ref DNRF123); People Programme (Marie Curie Actions FP7/2007-2013- n 609405).

\section{REFERENCES}

[1] S. Arnon et al., "Non-line-of-sight underwater optical wireless communication network", J. Opt. Soc. Am. A, 26, 3,(2009).

[2] V. Scarani et al. "The security of practical quantum key distribution", Rev. Mod. Phys. 81, (2009).

[3] C. Branciard, et al., "Upper bounds for the security of two distributedphase reference protocols of quantum cryptography", New Journal of Physics, Volume 10, (2008).

[4] D. Bacco et al., "Two-dimensional distributed-phase-reference protocol for quantum key distribution", Sci. Rep, 6, 36756, (2016)

[5] P. Shi, et al., "Channel analysis for single photon underwater free space quantum key distribution", J. Opt. Soc. Am. A, 32, (2015).

[6] J. Uhlmann, et al., "Quantum communications in the maritime environment," OCEANS 2015 - MTS/IEEE, Washington, DC, (2015)

[7] L. Ji et al., "Towards quantum communications in free-space seawater", Optics Express, 25.17: 19795-19806 (2017).

[8] F. Bouchard et al., "Underwater Quantum Key Distribution in Outdoor Conditions with Twisted Photons", arXiv: 1801.10299,(2018).

[9] H. Kaushal and G. Kaddoum, "Underwater Optical Wireless Communication," in IEEE Access, vol. 4, (2016). 